\title{
Phase I trial of the androgen receptor modulator CR1447 in breast cancer patients
}

\author{
Martin Zweifel' ${ }^{1}$, Beat Thürlimann², Salome Riniker², Patrik Weder' ${ }^{2}$, \\ Roger von Moos ${ }^{3}$, Olivia Pagani ${ }^{4}$, Martin Bigler ${ }^{5}$, Karin M Rothgiesser ${ }^{5}$, \\ Christiane Pilop ${ }^{5}$, Hanne Hawle ${ }^{5}$, Peter Brauchli ${ }^{5}$, Coya Tapia ${ }^{6}$, \\ Wolfgang Schoenfeld ${ }^{7}$ and Cristiana Sessa ${ }^{4}$ for the Swiss Group for Clinical Cancer \\ Research (SAKK)
}

1'Department of Medical Oncology, University Hospital Bern, Bern, Switzerland ${ }^{2}$ Breast Centre St. Gallen, Kantonsspital St. Gallen, St. Gallen, Switzerland ${ }^{3}$ Kantonsspital Graubünden, Chur, Switzerland

${ }^{4}$ Istituto Oncologico della Svizzera Italiana, Bellinzona, Switzerland

${ }^{5}$ SAKK Coordinating Centre, Bern, Switzerland

${ }^{6}$ Institute of Pathology, University of Bern, Bern, Switzerland

${ }^{7}$ CURADIS GmbH, Erlangen, Germany

Correspondence

should be addressed

to $\mathrm{M}$ Zweifel

Email

martin.zweifel@insel.ch

\begin{abstract}
CR1447 (4-hydroxytestosterone, 4-OHT) binds to the androgen receptor and has antiproliferative activity in both ER-positive and ER-negative/AR-positive breast cancer cells in preclinical studies. The objective of this first-in man trial was to evaluate the safety and to determine the dose of CR1447, administered as an ointment, for Phase II. Escalating doses $(100,200,400 \mathrm{mg})$ of CR 1447 were administered topically on a daily basis to patients with ER-positive/AR-positive/HER2-negative advanced breast cancer pretreated with several lines of therapy. 14 patients have been treated for a total of 42 cycles. Two patients, one at dose level $100 \mathrm{mg}$ and one at dose level $200 \mathrm{mg}$, showed early tumour progression and were replaced. Related adverse events were all $\leq$ grade 2 and included fatigue, bone and joint pain, stiffness, dry skin and mouth, nausea, sweating, urinary tract infection, rash, headache and distress. No drug-related dose-limiting toxicities (DLTs) were seen. Two patients (17\%) achieved stable disease at 3 months. Pharmacokinetic analysis confirmed dose-dependent transdermal uptake of CR1447. 4-OH-androstenedione (4-OHA), a key metabolite of 4-OHT, was undetectable in most of the plasma samples. Urine metabolites of 4-OHT and 4-OHA indicate high exposure of 4-OHT after topical administration. Oestradiol serum concentrations did not increase, confirming preclinical data that CR1447 is not converted to estrogens in vivo. In conclusion, CR1447 administered transdermally as an ointment is well tolerated and appears to have single-agent activity in heavily pretreated ER-positive/HER2-negative breast cancer patients. The recommended phase II dose is $400 \mathrm{mg} / \mathrm{day}$.
\end{abstract}

$\begin{array}{ll} & \text { Key Words } \\ \text { - } & \text { 4-OH-testosterone } \\ \text { - CR1447 } \\ \text { - androgen receptor } \\ \text { modulator } \\ \text { - breast cancer } \\ \text { - phase I trial }\end{array}$

Endocrine Connections (2017) 6, 549-556

\section{Introduction}

For the treatment of breast cancer (BC), testosterone was already used extensively between the 1930s and 1960s with anecdotal tumour responses (especially in bone metastases) seen in up to $20 \%$ of treated women $(1,2$, $3,4)$. Side effects such as hirsutism and other signs of virilisation in treated women and the evidence that 
testosterone may be easily converted to estrogens in the body led to the discontinuation of its use (5). These findings suggest that selective androgen receptor (AR) modulators (SARMs) rather than testosterone may be used to treat AR-positive BC.

CR1447 (4-hydroxytestosterone (4-OHT)) is a steroidal small molecule with two distinct properties, acting as a steroidal aromatase inhibitor (AI) and binding to the AR with high affinity ( $\left.\mathrm{IC}_{50} 4.4 \mathrm{nM}\right)$ (6). In vitro studies indicate that human BC cell lines are inhibited by CR1447 in their growth if they express the AR, while knockout of the AR abolishes this effect. A significant proportion of 4-OHT is converted to 4-hydroxyandrostenedione (4-OHA, formestane). In vivo, 4-OHT and 4-OHA form a redox reaction. 4-OHA is the 17-beta-oxidized isoform of CR1447. 4-OHA had previously been approved as an AI for the treatment of $\mathrm{BC}$ via intramuscular injection but was discontinued due to the development of oral AIs. Unlike testosterone, both compounds, 4-OHA and 4-OHT, are not converted to oestrogens in vivo $(7,8)$.

The combination of two mechanisms of action of CR1447 may result in higher activity. The objective of this phase I trial was to investigate the safety, tolerability, pharmacokinetics and the recommended dose (RD) of CR1447 for phase II in a clinical setting.

\section{Patients and methods}

\section{CR1447}

CR1447 was supplied in aluminium-coated stick packs as a $4 \mathrm{~g}$ ointment containing $2.5 \%$ of the active ingredient 4-hydroxytestosterone (4-OHT). CR1447 stick packs had to be stored protected from light at room temperature and should neither be refrigerated or frozen nor heated over $30^{\circ} \mathrm{C}$. CR1447 was supplied by CURADIS $\mathrm{GmbH}$, Erlangen, Germany and distributed by Promedipharm GmbH, Germany.

\section{Patients}

Eligible patients were postmenopausal women with locally advanced or metastatic, histologically confirmed breast adenocarcinoma requiring therapy and not suitable for local treatment. Inclusion criteria were immunohistochemical oestrogen receptor (ER) positivity $(\geq 1 \%$ ) and/or progesterone receptor (PR) positivity ( $\geq 1 \%$ ); in all cases, the tumour had to be human epidermal growth factor receptor 2 (HER2) negative. Presence of $\geq 1$ measurable or evaluable lesion according to RECIST 1.1 was required. Bone metastases were allowed to be considered as target lesions in case of enhancement on scintigraphy, cytologic/histologic evidence or typical radiographic image. Patients with uncontrolled brain metastases, pulmonary carcinomatous lymphangiosis or liver metastases on $>1 / 3$ of the liver were not eligible. All patients gave written informed consent after full explanation of the purpose and nature of all procedures applied.

\section{Study design and procedures}

This four centre (Bern University Hospital; Istituto Oncologico della Svizzera Italiana, Bellinzona; Kantonsspital St. Gallen; and Kantonsspital Graubünden, Chur, Switzerland), first-in man, open-label, phase I, dose escalation clinical trial (clinicaltrials.gov ID: NCT02067741) was reviewed by the Swiss Group for Clinical Cancer Research (SAKK) internal review process and approved by the Central and Local Ethics Committees and the Swiss Agency for Therapeutic Products (Swissmedic).

The main objective of the phase I was to determine safety, tolerability and the recommended dose (RD) of CR1447 for phase II, using a standard $3+3$ dose escalation design.

Treatment consisted of CR1447 (provided in 100mg stick packs) topically applied daily to the haunches and thighs until progression. One cycle was defined as lasting 21 days. Three dose escalation steps (100, 200 and $400 \mathrm{mg}$ daily) were performed including 3 patients in each dose cohort, with another 3 confirmatory patients in the last dose cohort or in case of a dose-limiting toxicity (DLT). A DLT was defined as an adverse event (AE), laboratory abnormality or a drug toxicity $\geq$ grade 3 that was considered to be probably, possibly or definitely related to the trial medication and which occurred during the first cycle of treatment. While 100 and $200 \mathrm{mg}$ were applied once daily, $400 \mathrm{mg}$ were applied as $200 \mathrm{mg}$ twice daily at the same body site. The planned sample size was between 2 and 18 evaluable patients. Since the overall toxicity of CR1447 was estimated to be low, the patients could switch to the next dose level when the next higher cohort of 3 patients had finished first cycle treatment without DLTs. The dose was escalated to the next higher dose level, whenever 0 out of 3 or $\leq 1$ out of 6 patients had a DLT. Patients were evaluated for DLT only at the lowest level 
they had been treated. If no DLTs were to occur at the highest dose level, this level was determined to be the recommended dose for phase 2 .

Dose modifications were planned for adverse events (AEs) grade 2 (dose reduction by one dose level) and grade 3 or 4 (stop treatment with CR1447 until resolved to grade $\leq 2$ and restart one dose level lower until end of treatment). If an $\mathrm{AE}$ grade 3 or 4 occurred on an already reduced dose, CR1447 had to be stopped until the AE resolved to grade $\leq 2$; treatment could then be resumed at one lower dose level.

Patients had to be taken off treatment if they missed $\geq 7$ days of treatment within the 1 st cycle or $>3$ weeks of treatment within subsequent cycles.

Secondary endpoints were disease control at 24 weeks (DC24) i.e. complete remission (CR), partial remission (PR) and stable disease (SD) within the first $24 \pm 2$ weeks of treatment or stable disease (SD) for at least 24 weeks after treatment start; AEs; pharmacokinetic (PK) analysis of CR1447; pharmacodynamic (PD) analysis i.e. oestradiol levels during treatment; AR and Ki67 expression. Disease control at 12 weeks (DC12) was included as an additional secondary endpoint later on.

\section{Pharmacokinetics (PK) and pharmacodynamics (PD)}

Plasma and serum 4-OHT, 4-OHA (for PK) and oestradiol (for PD) concentrations were measured at baseline, after single dose administration at 1, 2, 4, 8, 24 and $72 \mathrm{~h}$ (day $-7,-6,-5$ ) and during treatment at 1,8 and 15 days. Urine was collected from 0 to $8 \mathrm{~h}$, and from 8 till $24 \mathrm{~h}$ after single administration of CR1447. Urine 4-OHT, 4-OHA (for PK) and oestradiol (for PD) concentrations were assessed at baseline, once at week 2 and once at week 3 of treatment.

High-pressure liquid chromatography with mass spectrometry (HPLC-MS) was used for the determination of 4-OHT and 4-OHA in human plasma, with testosterone-d3 and exemestane being used as internal standards, respectively. The lower limit of quantification of each analyte in plasma was $0.25 \mathrm{ng} / \mathrm{mL}$.

\section{Assessment of androgen receptor status}

Central immunohistochemistry staining for the AR of the most recent formalin-fixed paraffin-embedded tissue samples was performed for all patients and scored semi-quantitatively. AR positivity was defined as any positive nuclear staining $\geq 1 \%$ in analogy to the College of American Pathologists' (CAP) recommendations for
(C) 2017 The authors

http://www.endocrineconnections.org DOI: 10.1530/EC-17-0174 reporting oestrogen receptor status (9). For every patient's tumour tissue sample, percentage of AR-positive tumour cell nuclei and staining intensity, semi-quantitatively scored from 1 to 3 , were determined.

\section{Assessment of response}

Best response was assessed by Response Evaluation Criteria in Solid Tumours (RECIST), version 1.1 (10). CT and bone scans were performed within 2 weeks prior to starting treatment at baseline, at week 12 ( \pm 8 days) and 24 ( \pm 2 weeks). In case of radiologic response (i.e. CR or PR), a confirmatory scan had to be performed after 4 weeks ( \pm 8 days).

\section{Data analysis and statistics}

The primary DLT and MTD analysis was performed on all evaluable patients who signed the informed consent and who did not miss $\geq 7$ days of treatment during the first cycle of treatment or had a DLT.

Other safety and efficacy data were analysed on all patients who received at least one dose of trial treatment.

Continuous data were summarised using median, minimum and maximum values. Categorical data were summarised using frequency and percentage.

Paired $t$ test was used to compare the body weights of patients at the time of screening vs at the end of treatment.

\section{Results}

Between June 2014 and February 2015, fourteen patients were recruited onto the trial. Patient characteristics are listed in Table 1. Most patients presented with bone metastasis and all patients had received prior endocrine therapy. Two patients (one in dose level 1 and one in dose level 2) were not evaluable: one patient had progressive disease after two weeks of treatment; therefore, she was taken off trial since she missed $\geq 7$ days of treatment. The other patient's cancer progressed after two weeks of treatment and was taken off trial. Twelve patients completed the first cycle of therapy, three patients each at dose level 1 and 2 and six (three plus three confirmatory patients) at dose level 3. All fourteen patients were used for the analysis of toxicity and efficacy data.

All patients' tumours stained positive for the AR (Table 2). Percentage of AR-positive nuclei ranged from 70 to $100 \%$ and staining intensity from 2 to 3 . 
Table 1 Patient characteristics for the evaluable patients.*

\begin{tabular}{lc}
\hline Characteristics & Count $(n=12)$ \\
\hline $\begin{array}{lc}\text { Age (years) } \\
\text { Median }\end{array}$ & 66 \\
$\quad$ Range & $44-80$ \\
WHO performance status & \\
0 & $8(67 \%)$ \\
1 & $4(33 \%)$ \\
Prior therapy & $10(83 \%)$ \\
$\quad$ Chemotherapy & $7(58 \%)$ \\
Radiotherapy & $12(100 \%)$ \\
Endocrine therapy & \\
Metastases & $11(92 \%)$ \\
Bone & $6(50 \%)$ \\
Liver & $6(50 \%)$ \\
Lymph nodes & $3(25 \%)$ \\
Lung & $2(17 \%)$ \\
Skin & $1(8 \%)$ \\
Brain & \\
\hline
\end{tabular}

*Two patients were not evaluable due to early progression within 2 weeks after the start of study treatment and had to be replaced.

Dose escalation and number of cycles administered are summarized in Table 3. Fourteen patients received a total of 42 cycles. Doses had to be modified in one cycle $(6 \%)$ in the dose level 1 group, in one cycle (20\%) in the dose level 2 group and in nine cycles (45\%) in the dose level 3 group. The only dose reduction for toxicity reasons was made in one patient in the dose level 3 group for dry skin. Dose modifications are listed in Table 4.

\section{Toxicity}

Overall, 92 AEs (including blood count and chemistry changes) grade $\geq 1$ according to NCI CTCAE, v4.0 occurred

Table 2 Androgen receptor status upon immunohistochemistry.

\begin{tabular}{|c|c|c|}
\hline Patient No. & AR positive nuclei (\%) & AR staining intensity score \\
\hline 1 & 100 & 3 \\
\hline 2 & 99 & 3 \\
\hline 3 & 100 & 3 \\
\hline 4 & 99 & 2 \\
\hline 5 & 95 & $2-3$ \\
\hline 6 & 95 & 2 \\
\hline 7 & 99 & 3 \\
\hline 8 & 70 & 3 \\
\hline 9 & 80 & 2 \\
\hline 10 & 95 & 3 \\
\hline 11 & 95 & 3 \\
\hline 12 & 100 & 3 \\
\hline 13 & 90 & 3 \\
\hline 14 & 95 & 3 \\
\hline
\end{tabular}

during treatment. The numbers of AEs per patient in the safety population that were possibly, probably or definitely drug related are summarised in Table 5.

Haematological toxicity was mild with only two patients experiencing grade $1 / 2$ thrombocytopenia and one patient with grade 1 neutropenia. Anaemia was the most common haematological $\mathrm{AE}$ in $50 \%$ of patients, of which all were grade $1 / 2$.

Elevated triglycerides were the most common blood chemistry AE in $57 \%$ of patients, of which all were grade $1 / 2$. Elevated liver function tests were seen in up to $50 \%$ of patients, including grade $3 / 4$ toxicity. Renal function tests were elevated in $29 \%$ of patients, of which all were grade $1 / 2$.

Drug-related non-haematological toxicity included rash and burning sensation of the skin in $14 \%$ of patients and dry skin in $21 \%$ patients. General symptoms included nausea and fatigue in $14 \%$ of patients each, sweating, headache, dry mouth, hot flashes and arthralgia in 7\% of patients each. Bone pain and decreased joint range of motion occurred in $14 \%$ of patients each. One patient experienced a urinary tract infection and one patient suffered from agitation. All non-haematological AEs were grade $1 / 2$. Occurrence of hirsutism was not observed. Body weight remained unchanged during treatment with CR1447. Mean body weight at screening was $69.36 \mathrm{~kg}$ (S.D. 15.93) compared to $69.71 \mathrm{~kg}$ (S.D. 15.34) at the end of treatment $(P=0.54)$.

Two serious adverse events (SAEs) were seen in this study. A 70-year-old patient in the dose level 2 group was admitted to the hospital with abdominal pain. Trial medication was discontinued and the patient died ten days later. Tumour progression was deemed the cause of death and the event was judged unrelated to the study medication. The second patient, a 54-year-old woman with brain metastases and a history of deep vein thrombosis, developed intracranial haemorrhage under anticoagulation with enoxaparin one month after starting on the trial. CR1447 was temporarily discontinued and enoxaparin was stopped. The patient recovered with residual symptoms of hemiplegia, but her tumour progressed and she was taken off trial. The event was judged unrelated to the study medication.

\section{Response}

Of the 14 patients, none achieved disease control (DC) at 24 weeks. Two (14\%) patients showed s.D. at 12 weeks; the first patient with S.D. at 12 weeks had previous treatment 
Table 3 Dose escalation and number of cycles administered.

\begin{tabular}{|c|c|c|c|}
\hline & \multicolumn{3}{|c|}{ All patients $(n=14)$} \\
\hline & DL 1 (100 mg) & DL 2 (200 mg) & DL 3 (400 mg) \\
\hline No. of cycles & 4 & 4 & 6 \\
\hline 1 & $1(25 \%)$ & $3(75 \%)$ & $1(17 \%)$ \\
\hline 2 & - & $1(25 \%)$ & $3(50 \%)$ \\
\hline 3 & - & - & - \\
\hline 4 & $2(50 \%)$ & - & - \\
\hline 5 & - & - & $1(17 \%)$ \\
\hline 6 & - & - & - \\
\hline 7 & - & - & - \\
\hline 8 & $1(25 \%)$ & - & $1(17 \%)$ \\
\hline
\end{tabular}

\begin{tabular}{|c|c|c|}
\hline \multicolumn{3}{|c|}{ Evaluable patients $(n=12)$} \\
\hline DL 1 (100 mg) & DL $2(200 \mathrm{mg})$ & DL $3(400 \mathrm{mg})$ \\
\hline 3 & 3 & 6 \\
\hline - & $2(67 \%)$ & $1(17 \%)$ \\
\hline - & $1(33 \%)$ & $3(50 \%)$ \\
\hline - & - & - \\
\hline $2(67 \%)$ & - & - \\
\hline- & - & $1(17 \%)$ \\
\hline - & - & - \\
\hline - & - & - \\
\hline 1 (33\%) & - & $1(17 \%)$ \\
\hline
\end{tabular}

with two lines of endocrine treatment (letrozole for 5 years in the adjuvant setting, followed by tamoxifen for 1 year in the palliative setting) and two lines of chemotherapy (cyclophosphamide, methotrexate and 5-fluorouracil, followed by epirubicin and cyclophosphamide); the second patient with s.D. at 12 weeks had two lines of endocrine treatment (tamoxifen for two years, followed by anastrozole for 7 months, both in the palliative setting). Eleven (79\%) patients showed progressive disease and $1(8 \%)$ patient's response could not be assessed due to withdrawal of consent beforehand.

\section{Pharmacokinetics}

A total of 140 study test samples were analysed in order to determine 4-OHT and 4-OHA plasma concentrations.

Plasma concentrations after single dose administration of $100 \mathrm{mg}$ CR1447 are shown in Fig. 1A. 4-OHT plasma concentrations were in the range of blq (below the lower limit of quantification) to $1.88 \mathrm{ng} / \mathrm{mL}$. The $C_{\max }$ and AUC values showed high inter-individual variability. Median $T_{\max }(n=12)$ for 4-OHT was $16 \mathrm{~h}$ (range: 1.0-72.0), median $C_{\max }(n=14)$ was $0.63 \mathrm{ng} / \mathrm{mL}$ (range: $0.0-1.88$ ) and the median AUC0-72 $(n=12)$ was $27.2 \mathrm{~h}^{*} \mathrm{ng} / \mathrm{mL}$ (range: 0.0-69.8).

The 4-OHA plasma concentrations were below the lower limit of quantification in all samples after single dose except for one sample tested.

Table 4 Dose deviations (all patients, $n=14$ ).

\begin{tabular}{|c|c|c|c|}
\hline & DL 1 & DL 2 & DL3 \\
\hline Cycles (n) & 17 & 5 & 20 \\
\hline No deviation & $16(94 \%)$ & $4(80 \%)$ & $11(55 \%)$ \\
\hline Physician's choice & - & - & $2(10 \%)$ \\
\hline Patient's choice & - & - & $6(30 \%)$ \\
\hline Toxicity & - & - & $1(5 \%)$ \\
\hline Other* & $1(6 \%)$ & $1(20 \%)$ & - \\
\hline
\end{tabular}

*Patients forgot taking the medication.
Plasma concentrations after multiple dose administration of CR1447 are shown for 4-OHT in Fig. 1B and for 4-OHA in Fig. 1C. 4-OHT plasma concentrations on day 8 and day 15 were in the range of $0.37-2.81 \mathrm{ng} / \mathrm{mL}$ for dose level $100 \mathrm{mg}, 0.72-5.61 \mathrm{ng} / \mathrm{mL}$ for dose level $200 \mathrm{mg}$ and $2.06-10.05 \mathrm{ng} / \mathrm{mL}$ for dose level $400 \mathrm{mg}$. 4-OHA plasma concentrations on day 8 and day 15 were in the range of blq to $0.34 \mathrm{ng} / \mathrm{mL}$ for dose level $100 \mathrm{mg}$, blq to $0.89 \mathrm{ng} / \mathrm{mL}$ for dose level $200 \mathrm{mg}$ and blq to $0.83 \mathrm{ng} / \mathrm{mL}$ for dose level $400 \mathrm{mg}$. 4-OHT and 4-OHA plasma concentrations increase with rising dose levels. No conclusions with regard to dose linearity can be drawn from these data, due to the high inter-individual variability and the limited number of data.

Table 5 Worst drug-related toxicity per patient (all cycles).

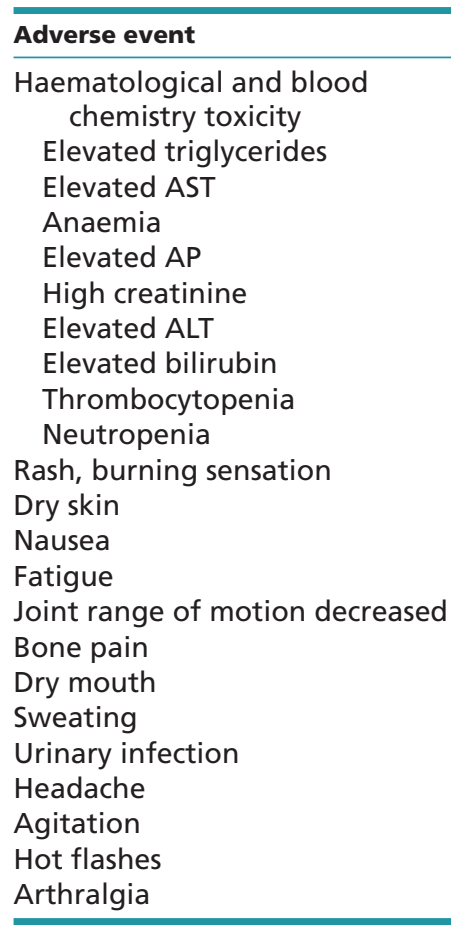

\begin{tabular}{|c|c|}
\hline Grade 1/2 & Grade $3 / 4$ \\
\hline $57 \%(8)$ & - \\
\hline $29 \%(4)$ & $21 \%(3)$ \\
\hline $50 \%(7)$ & - \\
\hline $29 \%(4)$ & $14 \%(2)$ \\
\hline $29 \%(4)$ & - \\
\hline $21 \%(3)$ & $7 \%(1)$ \\
\hline $7 \%(1)$ & $14 \%(2)$ \\
\hline $21 \%(3)$ & - \\
\hline $7 \%(1)$ & - \\
\hline $21 \%(3)$ & - \\
\hline $21 \%(3)$ & - \\
\hline $14 \%(2)$ & - \\
\hline $14 \%(2)$ & - \\
\hline $14 \%(2)$ & - \\
\hline $7 \%(1)$ & - \\
\hline $7 \%(1)$ & - \\
\hline $7 \%(1)$ & - \\
\hline $7 \%(1)$ & - \\
\hline $7 \%(1)$ & - \\
\hline $7 \%(1)$ & - \\
\hline $7 \%(1)$ & - \\
\hline $7 \%(1)$ & - \\
\hline
\end{tabular}

This work is licensed under a Creative Commons Attribution-NonCommercial-NoDerivatives 4.0 International License. 
reduction of the antiproliferative effects of 4-OHT (11). In this first-in man phase I trial, we investigated the toxicity, pharmacokinetics and pharmacodynamics of CR1447 in patients with ER-positive/AR-positive/HER2-negative BC.

Toxicity in general was mild. Anaemia was the most common haematological toxicity, while blood chemistry changes were observed mostly for triglycerides, liver and kidney function tests. While six out of seven patients who experienced liver and kidney function test impairment also suffered from liver metastasis, the finding of altered lipid profiles is not surprising. Based on previous experience with AIs, one might assume that CR1447 with its known AI activity may also elicit similar side effects (12). However, none of those led to dose modifications or disruptions regarding the treatment with CR1447 in our study. There is some controversy on the impact of AIs on the lipid profile. Lipid profile changes will need attending in future trials with CR1447.

Non-haematological toxicities included skin reactions/dry skin, which lead to a dose reduction in one patient only. These symptoms and other, less frequent ones such as nausea, fatigue, sweating, headache, bone and joint pain, dry mouth and hot flashes might fit well to the antihormonal effects of CR1447. All drug-related non-haematological toxicities were of grade $1 / 2$ only.

The two serious adverse events (SAEs) seen in this study (death due to tumour progression and intracranial haemorrhage under anticoagulation) were judged unrelated to the study medication. No DLTs were seen.

The observation of two patients (17\%) with stable disease after 12 weeks of treatment suggests some singleagent activity, given that all patients received prior endocrine therapy, and $83 \%$ of these patients also had prior chemotherapy.

Recently, there has been a growing interest in targeting the AR in the treatment of BC. Clinical trials report a median progression-free survival of 12 weeks with the non-steroidal AR antagonists bicalutamide (13) and orteronel (14), but even complete responses have been observed (15). In a combination trial of the nonsteroidal AR antagonist enzalutamide with the oestrogen antagonist fulvestrant, $45 \%$ of patients still remained on treatment after 24 weeks (16). The non-steroidal AR modulator GTX-024 demonstrated clinical benefit in 35\% of patients with metastatic BC at 6 months (17). Disease control at 12 weeks in our study is comparable with the results of the other studies cited. However, there are concerns that a pure antagonist could foster virilisation. Therefore, a partial antagonist, or selective androgen receptor modulator (SARM) such as the steroidal AR antagonist CR1447 might offer an advantage over the non-steroidal AR antagonists.

PK results confirmed a relatively quick absorption of 4-OHT. One could have speculated that 4-OHT might be rapidly converted to 4-OHA in vivo, a drug which has been used previously as an AI (formestane, Lentaron). Formestane had to be given by intramuscular injection because of fast metabolisation if taken orally (18). Interestingly, our PK data show that the ratio of 4-OHT:4-OHA in the serum is roughly 10:1 even after two weeks of daily administration. However, the ratio of 4-OHT:4-OHA in urine is 1:5, possibly indicating metabolisation of $4-\mathrm{OHT}$ to $4-\mathrm{OHA}$ prior to and/or during elimination.

Serum oestradiol concentrations did not change during treatment with CR1447, indicating no relevant conversion of the drug to oestrogen.

The dose recommended for phase 2 trials must take account of the RECIST and toxicity data. As, due to the steroidal nature of the molecule, we did not anticipate issues with dose-related toxicity, we chose three different dose levels on the basis of preclinical studies and extrapolations from previous experience with 4-OHA. A relatively high number of cycles could be evaluated for toxicity (16 in DL1 vs 4 in DL 2 and 20 in DL3). CR1447 was well tolerated at all dose levels, including the highest dose level at $400 \mathrm{mg} /$ day.

In summary, CR1447 administered transdermally as an ointment was well tolerated and appears to have single-agent activity in heavily pretreated ER-positive/ AR-positive/HER2-negative BC patients. The next step will be a phase II trial in order to test the hypothesis if CR1447 might offer the possibility of non-chemotherapybased endocrine therapy to the limited treatment options in triple-negative (TN)/AR-positive BC. The recommended phase II dose is $400 \mathrm{mg} /$ day.

\section{Declaration of interest}

W S is employed by CURADIS GmbH. The authors would like to confirm that there are no other known conflicts of interest associated with this publication for all other co-authors.

\section{Funding}

This work was supported by the Swiss State Secretariat for Education, Research and Innovation, and by CURADIS GmbH, Erlangen, Germany.

\section{Acknowledgements}

For protocol development, M Z was supported by the 15th ECCO-AACREORTC-ESMO Workshop on Methods in Clinical Cancer Research, Flims, Switzerland, 22nd-28th June 2013. This work was presented at the 38th San Antonio Breast Cancer Symposium (SABCS), December 8-12, 2015, San Antonio, Texas, USA. 


\section{References}

1 Fels E. Treatment of breast cancer with testosterone propionate. A preliminary report. Journal of Clinical Endocrinology 19444 121-125. (doi:10.1210/jcem-4-3-121)

2 Goldenberg IS. Testosterone propionate therapy in breast cancer. JAMA 1964188 1069-1072. (doi:10.1001/ jama.1964.03060380037009)

3 Segaloff A, Gordon D, Horwitt BN, Schlosser JV \& Murison PJ. Hormonal therapy in cancer of the breast. I. The effect of testosterone propionate therapy on clinical course and hormonal excretion. Cancer 19514 319-323. (doi:10.1002/1097-0142(195103)4:2<319::AIDCNCR2820040217>3.0.CO;2-5)

4 Segaloff A, Weeth JB, Cuningham M \& Meyer KK. Hormonal therapy in cancer of the breast. 23. Effect of 7-alpha-methyl19-nortestosterone acetate and testosterone propionate on clinical course and hormonal excretion. Cancer 196417 1248-1253. (doi:10.1002/1097-0142(196410)17:10<1248::AIDCNCR2820171005>3.0.CO;2-A)

5 Malarkey WB, Schroeder LL, Stevens VC, James AG \& Lanese RR. Twenty-four-hour preoperative endocrine profiles in women with benign and malignant breast disease. Cancer Research 197737 4655-4659. (doi:10.1186/bcr2931)

6 Miller WR \& Larionov AA. Understanding the mechanisms of aromatase inhibitor resistance. Breast Cancer Research 201214201. (doi:10.1186/bcr2931)

7 Johannessen DC, Adlercreutz H, Fotsis T \& Lonning PE. Plasma and urinary oestrogens in breast cancer patients on treatment with 4-hydroxyandrostenedione. British Journal of Cancer 199368 393-398. (doi:10.1038/bjc.1993.347)

8 Kohler M, Parr MK, Opfermann G, Thevis M, Schlorer N, Marner FJ $\&$ Schanzer W. Metabolism of 4-hydroxyandrostenedione and 4-hydroxytestosterone: mass spectrometric identification of urinary metabolites. Steroids 200772 278-286. (doi:10.1016/j. steroids.2006.11.018)

9 Hammond ME. Commentary: improving breast cancer testing for patients-the secret sauce is collaboration. Journal of Oncological Practices 20106 198. (doi:10.1200/JOP.777012)

10 Eisenhauer EA, Therasse P, Bogaerts J, Schwartz LH, Sargent D, Ford R, Dancey J, Arbuck S, Gwyther S, Mooney M, et al. New response evaluation criteria in solid tumours: revised RECIST guideline (version 1.1). European Journal of Cancer 200945 228-247. (doi:10.1016/j. ејса.2008.10.026)
11 Schoenfeld W. CR1447 Investigator's Brochure, Edition No. 3. Erlangen, Germany: Curadis GmbH, 2017. (available at: http://www. curadis.eu/forschung-entwicklung/cr-1447/)

12 Gonnelli S \& Petrioli R. Aromatase inhibitors, efficacy and metabolic risk in the treatment of postmenopausal women with early breast cancer. Clinical Interventions in Aging 20083 647-657. (doi:10.2147/ CIA.S3466)

13 Gucalp A, Tolaney S, Isakoff SJ, Ingle JN, Liu MC, Carey LA, Blackwell K, Rugo H, Nabell L, Forero A, et al. Phase II trial of bicalutamide in patients with androgen receptor-positive, estrogen receptor-negative metastatic breast cancer. Clinical Cancer Research 201319 5505-5512. (doi:10.1158/1078-0432.CCR-12-3327)

14 Yardley DY, Peacock N, Young R, Silber A, Chung G, Webb C, Jones SF, Shastry M, Midha R, DeBusk L, et al. A Phase II study evaluating orteronel, an inhibitor of androgen biosynthesis, in patients with androgen receptor (AR)-expressing MBC-interim analysis of the ER+ and/or PR+ cohort. Presented at the San Antonio Breast Cancer Symposium 2015. Cancer Research 201676 (Supplement 4) abstract P5-14-04. (doi:10.1158/1538-7445.SABCS15-P5-14-04)

15 Arce-Salinas C, Riesco-Martinez MC, Hanna W, Bedard P \& Warner E. Complete response of metastatic androgen receptor-positive breas cancer to bicalutamide: case report and review of the literature. Journal of Clinical Oncology 201634 e21-e24. (doi:10.1200/ JCO.2013.49.8899)

16 Elias AD, Burris HA, Patel MR, Schwartzberg LS, Richer JK, Kavalerchik E, Stopatschinskaja S, Gibbons J, Markova D, Steinberg JL, et al. MDV310008: a phase 1 study evaluating the safety and pharmacokinetics of enzalutamide plus fulvestrant in women with advanced hormone receptor-positive breast cancer. Presented at the San Antonio Breast Cancer Symposium 2015. Cancer Research 201676 (Supplement 4) abstract P1-16-05. (doi:10.1158/1538-7445.SABCS15-P1-16-05)

17 Overmoyer B, Rugo H, Schwartzberg LS, Palmieri C, Taylor R, Hancock M, Small S \& Johnston MA. Phase 2 open label, multinational, randomized, parallel design study investigating the efficacy and safety of GTx-024 on metastatic (MET) or locally advanced (LA) ER+/AR+ breast cancer (BC) in postmenopausal (PM) women. Presented at the San Antonio Breast Cancer Symposium 2015. Cancer Research 201676 (Supplement 4) abstract OT2-01-06. (doi:10.1158/1538-7445.SABCS15-OT2-01-06)

18 Coombes RC. 4-Hydroxyandrostenedione treatment for postmenopausal patients with breast cancer. Journal of Steroid Biochemistry and Molecular Biology 199243 145-148. (doi:10.1016/0960-0760(92)90199-S)

Received in final form $\mathbf{3 1}$ July 2017

Accepted 16 August 2017

Accepted Preprint published online 16 August 2017 http://www.endocrineconnections.org DOI: 10.1530/EC-17-0174 (c) 2017 The authors Published by Bioscientifica Ltd

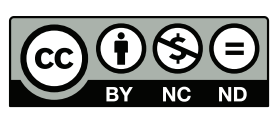

This work is licensed under a Creative Commons Attribution-NonCommercial-NoDerivatives 4.0 International License. 